The Horticultural Notebook. Compiled. by J. C. Newsham. Third edition, thoroughly revised. Pp. $x x+418$. (London: Crosby Lockwood and Son, 1914.) Price $4 s .6 d$. net.

THE fact that this work of reference has reached its third edition, and that its price has been reduced, proves that its usefulness is now generally recognised. It is, indeed, a book of convenient size and shape, which anyone whose interests are largely bound up in horticulture will find useful to have on his writing-table. As everyone knows who follows this pursuit, minor problems are cropping up almost every day of one's life. The strength of an insecticide or a manure,-some simple way of ascertaining the height of a tree without climbing it, the right dimensions of a lawn tennis court, how to make a grafting wax : these are samples of the kind of question for which those concerned with gardens are constantly needing an answer. This the "Horticultural NoteBook" sets out to supply, and we do not find that it often fails.

Although the serious student will need something more detailed than is here furnished, the book is not devoid of scientific teaching. A synopsis of the natural orders of plants, for instance, is concisely and conveniently arranged and helpful in "running down" a plant. It is not, however, in this direction (which suggests too much a shilling encyclopedia) that the value of the book consists, so much as in the collection of garden recipes and rules, and in much tabulated information. The ancient and remarkably persistent error that the plane tree of the streets is the American Platanus occidentalis is once more repeated here (p. 368), although it has several times been pointed out in these columns that the tree is really the Old World $P$. acerifolia.

Ornamental Lathework for Amateurs. By C. H. C. Pp. r2 I + xii plates. (London: Percival Marshall and Co., n.d.) Price $3 s .6 d$. net.

Plain turning is carried out in an ordinary lathe by revolving the work and operating on it by tools held in the hand or in a slide-rest. In ornamental turning, an object already subjected to plain turning processes is ornamented by further operations carried out on it by cutters which are made to revolve independently of the lathe mandrel. Ornamental turning is an exceedingly beautiful art, and the object of the little book before us is to awaken the interest of those who have adopted turning as a hobby, and to show how simply an ordinary turning lathe may be modified so as to be capable of producing beautiful examples of ornamental turning. While many examples are given and illustrated by photographs, it is not the author's intention that these should be used as designs to be worked out, but rather to stimulate the worker to devise new designs and methods for himself. Drawings of many useful types of tools are given, mostly of a simple character. The book can be recommended as a useful introduction to any amateur turner who has not yet taken up this fascinating branch of his art.

No. 2335, voL. 93]

\author{
LETTERS TO THE EDITOR.
}

[The Editor does not hold himself responsible for opinions expressed by his correspondents. Neither can he undertake to return, or to correspond with the irriters of, rejected manuscripts intended for this or any other part of NATURE. No notice is taken of anonymous communications.]

\section{Rayleigh's Law of Extinction and the Quantum Hypothesis.}

THE bearing of Rayleigh's law of gaseous extinction on some of the fundamental aspects of radiation theory does not seem to have been sufficiently emphasised in recent reports and publications on modern molecular physics. The coefficient of attenuation $\kappa$ of radiation of wave-length $\lambda$ travelling through a gas containing $n_{0}$ molecules per unit volume was given by Rayleigh ${ }^{2}$ so long ago as I87I in the form $k=\pi^{3}\left(\mu_{0}^{2}-1\right)^{2} \lambda^{-4} / n_{0}$, $\mu_{0}$ being the refractive index of the gas. It is of importance to notice that the law in question is one of the most fundamental results of molecular dynamics, its final expression being an invariant with respect to the theories of the æether or of the molecule employed, ${ }^{2}$ while in its derivation there is no need to draw on resources outside classical dynamics and continuous energy-flow. From the point of view of elementary electromagnetic theory, the above expression for $k$ is very easily derived along lines suggested in a problem set in part ii. of the "Mathematical Tripos" "3 use is made of the conventional electrical doublet set into forced vibrations by a train of electromagnetic waves; by making use of the radiation formula for accelerated charges and Poynting's theorem, the flow of energy from the doublet is easily calculated in terms of the amplitude of vibration; the oscillations of the doublet contribute a term to Maxwell's displacement current, enabling the amplitude to be expressed in terms of the refractive index of the gas; by considering the depletion of energy from the original beam as a result of this scattering, and eliminating the amplitude, the above expression for $\kappa$ is easily obtained. In a recent paper, Natanson " has subjected the derivation of Rayleigh's law to minute criticism on the grounds of the classical electromagnetic theory, allowing for a damping term arising from the mechanical reaction due to radiation, and taking into special consideration the summation of the aggregate radiation from the random distribution of doublets which are supposed to constitute the molecules of the gas; the final result is a vindication of the above expression for the coefficient of attenuation to a very high order of accuracy. It may be noticed in passing that the same electro. magnetic system forms the basis of Planck's ${ }^{5}$ theory of "black-body" radiation, the interpretation of experiment in this case, however, necessitating the hypothesis of discontinucus energy-flow, or the emission of energy by "quanta."

For an adequate experimental verification of Rayleigh's law recourse must be had to observations on the extinction of solar radiation of different wavelengths by the earth's atmosphere. The importance of the observations of the Smithsonian Astrophysical Observatory on atmospheric transmission recently carried out by Abbot and Fowle ${ }^{6}$ in connection with their determinations of the solar constant at Mount Wilson, in furnishing material for a study of mole-

i Rayleigh, Phil. Mag., xli., pp. 107, 274, 447 (187r); "Collected Works," i., pp. 87, ro4, $5 \times 8$.

2 Schuster, "Theory of Optics," and ed. (rgog), p. 325

3 Mathematical Tripos, Part ii., lune 2 , 1906.

4 Natanson, Bull. litter. de l'Académie des Sciences de Cracovie January 5, xgr 4 .

5 Planck, "Thenry of Heat Radiation" (Trans. by Masius, Blakiston's, Philadelphia, 19r4). part iv., chap. iii. p $x 65$.

6 Annals of the Smithsonian Astrophysical Observatory. Washington : vol. ii. (rgo8); vol. iii. (1913). 